\title{
Thermal analysis of hadron multiplicities from relativistic quantum molecular dynamics
}

\author{
J. Sollfrank and U. Heinz \\ Institut für Theoretische Physik, D-93040 Universität Regensburg, Germany \\ H. Sorge \\ Department of Physics, State University of New York at Stony Brook, Stony Brook, New York 11794 \\ N. Xu \\ Lawrence Berkeley Laboratory, Berkeley, California 94720
}

\begin{abstract}
Some questions arising in the application of the thermal model to hadron production in heavy ion collisions are studied. We do so by applying the thermal model of hadron production to particle yields calculated by the microscopic transport model RQMD(version 2.3). We study the bias of incomplete information about the final hadronic state on the extraction of thermal parameters. It is found that the subset of particles measured typically in the experiments looks more thermal than the complete set of stable particles. The hadrons which show the largest deviations from thermal behavior in RQMD(version 2.3) are the multistrange baryons and antibaryons. We also looked at the influence of rapidity cuts on the extraction of thermal parameters and found that they lead to different thermal parameters and larger disagreement between the RQMD yields and the thermal model.
\end{abstract}

PACS number(s): 25.75.Dw,24.10.Lx,24.10.Pa

\section{INTRODUCTION}

The hadron production in nuclear collisions at relativistic and ultrarelativistic energies is under extensive experimental and theoretical investigation [1]. One question of debate is whether hadron production is thermal, i.e., whether the hadron multiplicities for a given volume and energy density are distributed according to maximum entropy, or whether the particle multiplicity distribution reflects additional dynamical constraints.

This question is not easy to answer. First the thermal model has various versions and depending on the additional input the answers on thermal parameters differ [2]. Some of the differences in the various models are the number of included resonance states [3], the use of a canonical or grand canonical framework 迎, 5, the inclusion of hard core repulsion [6], the inclusion of strangeness suppression [7], the inclusion of other offequilibrium parametrizations [8], or the inclusion of finite size corrections in the density of states [9].

The second important source of uncertainty in the judgment of thermal behavior is the incomplete measurement of the final hadronic state. Not all particles which are stable with respect to the strong interaction can be measured, and all measurements cover only a finite part of the complete momentum space. Some measurements cover nearly the full solid angle, e.g., NA49 [10], in symmetric collision systems, but some are restricted to only a small kinematic window 11$]$ 13].

The bias of the restricted information is the goal of our study here. We use the transport approach RQMD(version 2.3) 14 for generating the full momentum space information on all stable particles. RQMD provides a microscopic description of heavy ion collisions which has been quite successful in predicting most of the observed features over a wide range of conditions and is therefore very well suited for our investigations.

\section{THERMAL MODEL}

The parameters of the thermal model may be understood as Lagrangian multipliers in extremizing the total entropy under certain constraints. For a given point particle volume $V$ we have as parameters the temperature $T$ (the Lagrangian multiplier to achieve a certain average energy density) and chemical potentials $\mu_{\mathcal{Q}}$ (the Lagrangian multipliers to constrain the system on average to a conserved total charge $\mathcal{Q}$ ). In our case the conserved charges are baryon number $B$, strangeness $S$, and electrical charge $Q$. Instead of $B, Q$, and $S$ one can equivalently conserve the net number of valence quarks for each flavor, $\langle u-\bar{u}\rangle,\langle d-\bar{d}\rangle,\langle s-\bar{s}\rangle$, since on the relevant time scale only strong interactions are important. The relations between the chemical potentials in both descriptions are

$$
\begin{aligned}
& \mu_{B}=\mu_{u}+2 \mu_{d}, \\
& \mu_{Q}=\mu_{u}-\mu_{d}, \\
& \mu_{S}=\mu_{d}-\mu_{s} .
\end{aligned}
$$

The corresponding fugacities are defined by

$$
\lambda_{f}=e^{\mu_{f} / T}, \quad f=u, d, s .
$$

We know from pp collisions that strangeness is not in full chemical equilibrium with the nonstrange hadrons 
[15. Therefore we allow for a deviation of strange particles from complete chemical equilibrium. However, we force the strange particles to be in chemical equilibrium among each other [7]. This requirement is equivalent to the introduction of the additional constraint that the total number of strange valence quarks $\langle s+\bar{s}\rangle$ be conserved on the average. The Lagrangian multiplier for this constraint - expressed as a fugacity — is [16]

$$
\gamma_{s}=\exp \left(\mu_{\langle s+\bar{s}\rangle} / T\right) \text {. }
$$

$\gamma_{s}$ is a measure of the chemical saturation of strangeness with $\gamma_{s}<1$ meaning undersaturation, $\gamma_{s}=1$ saturation, i.e., chemical equilibrium, and $\gamma_{s}>1$ oversaturation.

In the grand canonical description with the parameters introduced above the multiplicity $N_{i}$ of a particle $i$ with mass $m_{i}$ and spin degeneracy $J_{i}$ is given by

$$
\begin{aligned}
N_{i}= & \left(2 J_{i}+1\right) \frac{V T}{2 \pi^{2}} \sum_{j=1}^{\infty}(\mp 1)^{j+1} \\
& \times\left(\gamma_{s}^{\langle s+\bar{s}\rangle_{i}} \lambda_{u}^{\langle u-\bar{u}\rangle_{i}} \lambda_{d}^{\langle d-\bar{d}\rangle_{i}} \lambda_{s}^{\langle s-\bar{s}\rangle_{i}}\right)^{j} \\
& \times \int_{-\infty}^{\infty} d m^{2} \delta\left(m^{2}-m_{i}^{2}\right) \theta(m) \frac{m^{2}}{j} K_{2}\left(\frac{j m}{T}\right) .
\end{aligned}
$$

The $\langle\ldots\rangle_{i}$ denote the corresponding valence quark contents of particle species $i$. The upper sign is for fermions, the lower sign for bosons. With the exception of pions we use for all particles the Boltzmann approximation; i.e., the sum over the index $j$ is truncated at $j=1$.

For broad resonances $(\Gamma>1 \mathrm{MeV})$ we replace the $\delta$ function in Eq. (1) by a Breit-Wigner distribution $\delta_{\mathrm{BW}}\left(m^{2}\right)$

$$
\begin{aligned}
& \delta\left(m^{2}-m_{i}^{2}\right) \theta(m) \longrightarrow \delta_{\mathrm{BW}}(m) \\
& \quad=\frac{\alpha m_{i} \Gamma_{i}}{\left(m^{2}-m_{i}^{2}\right)^{2}+m_{i}^{2} \Gamma_{i}^{2}} \theta\left(m-m_{\mathrm{thres}}\right) \theta\left(m_{\mathrm{cut}}-m\right),
\end{aligned}
$$

where $\Gamma_{i}$ is the width of the resonance $i, m_{\text {thres }}$ a threshold for the production of resonances, and $m_{\text {cut }}$ an upper cutoff. We use $m_{\text {thres }}=m_{i}-2 \Gamma_{i}$ and $m_{\text {cut }}=m_{i}+2 \Gamma_{i}$. The normalization $\alpha$ is determined by $\int d m^{2} \delta_{\mathrm{BW}}\left(m^{2}\right)=$ 1.

Equation (4) gives the multiplicity of primary hadrons and resonances. The index for the particle species $i$ runs over all hadrons of the Review of Particle Properties [17] with $m_{i} \leq 1.7 \mathrm{GeV}$. The final hadron yields of stable hadrons include the decay contributions of the resonances. Unless otherwise stated we consider all these hadrons as stable which are stable with respect to strong decays. An exception is the $\phi$ which is taken to be stable in order to be in agreement with the special mode of RQMD used here (see below). The branching ratios are taken from [17], where missing or inaccurate entries have been completed by the requirement that all branching ratios for a resonance have to add to 1 .
The thermal model used here is in practical respects very similar to the one in 55. The main difference is the use of the grand canonical approach for all conserved charges in the study here. In addition there are small differences in the implementation of particle properties and decays where accurate numbers are missing in [17. We use the following values for the assignment of hidden strangeness:

$$
\begin{aligned}
& \langle s+\bar{s}\rangle_{\eta}=\langle s+\bar{s}\rangle_{\eta^{\prime}}=1, \\
& \langle s+\bar{s}\rangle_{\phi}=\langle s+\bar{s}\rangle_{f_{0}(980)}=\langle s+\bar{s}\rangle_{f_{1}(1420)}=2, \\
& \langle s+\bar{s}\rangle_{f_{1}(1510)}=\langle s+\bar{s}\rangle_{f_{2}^{\prime}(1525)}=\langle s+\bar{s}\rangle_{\phi(1680)}=2 .
\end{aligned}
$$

We show in Tables and II a comparison between the fits using the fully grand canonical approach and the partly canonical approach of [5]. The two additional chemical potentials in our approach are determined by the same constraints as used in [5], i.e., strangeness neutrality and electric charge conservation:

$$
\begin{aligned}
& \sum_{i} S_{i} N_{i}=0 \\
& \sum_{i} Q_{i} N_{i}=\frac{Z}{A} \sum_{i} B_{i} N_{i} .
\end{aligned}
$$

$Z / A$ is the ratio of charge over mass number of the colliding nuclei. We have the same number of degrees of freedom as in the canonical approach. We see from Tables [1] and II that the two approaches are equivalent on the level of a few percent.

\section{THERMAL FITS TO $4 \pi$ MULTIPLICITIES}

We now turn to RQMD(version 2.3) as the source for our particle multiplicities. The RQMD transport model [14] is based on the excitation and fragmentation of colour strings followed by hadronic reinteractions. The version used [RQMD(version 2.3)] contains the color rope mechanism [25], an important source for the production of the strange hadrons which are of special interest in our study. It has been shown by comparison with experimental data 26,27] that RQMD reproduces the main properties in hadron production. It is therefore a reliable model for generating the full momentum space information of all produced stable hadrons. In order to get also a $\phi$ multiplicity we treated in this investigation the $\phi$ as a stable particle.

We generated about 400 RQMD events each for $\mathrm{S}+\mathrm{S}$ and $\mathrm{Pb}+\mathrm{Pb}$ collisions with a centrality of $5 \%$ of $\sigma_{\text {inel }}^{\text {tot }}$. The error on the input data is very important in the minimization of $\chi^{2}$. Hadron yields from RQMD have only a statistical error which can be made arbitrarily small if only enough events are generated. In order to put thermal fits to experimental data and thermal fits to RQMD yields on the same footing we give the multiplicities calculated by RQMD the same relative error as the 
corresponding experimental value. The actual statistical errors from 400 RQMD events were in nearly all cases smaller than the corresponding experimental ones. We show in Table III a summary of relative errors used for RQMD multiplicities, either taken directly from experiment or related to other entries of experimental relative errors.

In Tables IV and V (rightmost column) the $4 \pi$ yields of the RQMD(version 2.3) simulation are shown as well as yields counted in a central rapidity window of varying size. The given multiplicities do not contain any decay contributions from weak or electromagnetic decays. The cited RQMD multiplicities differ in this respect from the experimental ones. In the thermal fits the corresponding changes have always been incorporated by the use of the appropriate decay channels.

We performed different thermal fits to the RQMD $4 \pi$ multiplicities. First, we included in the thermal fit only the subset of particles as used in the thermal model description of experimental data in [5]. The result is shown in Table V1. Compared to the thermal fit of experimental data in 50 (see also Table [1] we see that the thermal fit parameters of the RQMD analysis are close to the experimental ones only in the case of $\mathrm{S}+\mathrm{S}$ collisions. A significant discrepancy is seen for the $\mathrm{Pb}+\mathrm{Pb}$ collisions. The extracted temperature for example differs by about $30 \mathrm{MeV}$. We interpret this as an indication of larger differences in the hadron production between RQMD and the experiment. We do not try to investigate where the main differences between RQMD and the data lie, since this is, first of all, out of scope of this paper and, second, most of the experimental data are preliminary and thus it is too premature to speculate about physical reasons. The reader should further note that a direct comparison of experimental yields and the here calculated yields from RQMD is not reasonable since the yields of $K, \Lambda$, and $\bar{\Lambda}$ contain no weak or electromagnetic contribution in the case of RQMD while such decays are partly included in the data. In addition, the experimental data of $\mathrm{Pb}+\mathrm{Pb}$ collisions contain hadron yields and ratios which were measured in a limited acceptance and were extrapolated to $4 \pi$. For all these reasons a thermal fit to the experimental $\mathrm{Pb}+\mathrm{Pb}$ data would be premature.

We would also like to draw the attention to the very low $\chi^{2} / \mathrm{DOF}$ compared to the experimental fit. The small value of $\chi^{2} / \mathrm{DOF}$ is partly a result of the used relative errors for RQMD. Since this error was taken from experiment, it contains the systematic error of the corresponding experiment. As a result of "perfect reconstruction efficiency" in RQMD the error is overestimated. Using only the statistical error of RQMD would lead to a $\chi^{2} / \mathrm{DOF}=2.37 / 4$ for $\mathrm{S}+\mathrm{S}$ collisions and $\chi^{2} / \mathrm{DOF}=28.00 / 3$ for $\mathrm{Pb}+\mathrm{Pb}$ collisions. Nevertheless, we keep the relative experimental error for RQMD yields, since we want to have the same relative weights of hadrons in a thermal fit as it is typical for an analysis of experimental data. We discuss the thermal character of RQMD yields in more detail below.
We now show in the rightmost column of Tables VII and VIII the resulting thermal parameters of a thermal fit including all stable particles from RQMD. The comparison between the RQMD yields and the thermal ones is given in Tables IV and V, respectively. The most striking difference to the fit with the reduced number of hadrons is the increase in the $\chi^{2} / \mathrm{DOF}$ by a factor of about 25 , while the thermal parameters are basically the same. Only for $\gamma_{s}$ do we observe a small decrease with increasing number of stable particles. The result is understood by looking at the largest deviations of the thermal fit from the RQMD yields in Tables [V] and V. In terms of standard deviations (s.d.) between the $4 \pi$ thermal fit and RQMD we have the largest deviations for $\Omega(\mathrm{S}+\mathrm{S}, 29$ s.d.; $\mathrm{Pb}+\mathrm{Pb}$, 15 s.d.), $\bar{\Omega}(\mathrm{S}+\mathrm{S}, 9$ s.d.; $\mathrm{Pb}+\mathrm{Pb}, 8$ s.d. $), \phi(\mathrm{S}+\mathrm{S}, 6.7$ s.d.; $\mathrm{Pb}+\mathrm{Pb}, 4.7$ s.d.) and $\mathrm{K}^{+}(\mathrm{S}+\mathrm{S}, 9$ s.d. $)$. Interestingly, also in the thermal fit of Ref. 8 to the $\mathrm{Pb}+\mathrm{Pb}$ data the $\bar{\Omega}$ and $\Omega$ spoil the otherwise good fit, although their deviations go into the opposite direction.

The above result shows that the thermal fit gets worse if all stable particles are considered, while a reduced number of particle species suggest a better thermal behavior. The multistrange baryons are mostly responsible for the breakdown of the otherwise nice thermal behavior. This is illustrated in Figs. 11 and 2 where the result of the thermal fit with the reduced number of input particles of Table VI is displayed. In addition to the fitted input particles (solid squares) the resulting other multiplicities of these thermal fits are shown as open circles. For both collision systems the $\Omega, \bar{\Omega}$, and $\Xi$ show the largest deviations. The $\chi^{2} / \mathrm{DOF}$ of the thermal model with the parameters of Table VI applied to all stable multiplicities of Tables IV and V. respectively, leads to $\chi^{2} / \mathrm{DOF}=224 / 21$ for $\mathrm{S}+\mathrm{S}$ and $\chi^{2} / \mathrm{DOF}=191 / 21$ for $\mathrm{Pb}+\mathrm{Pb}$, while excluding $\Omega, \bar{\Omega}, \Xi^{0}, \Xi^{-}, \bar{\Xi}^{0}$, and $\bar{\Xi}^{-}$leads to $\chi^{2} / \mathrm{DOF}=8.5 / 15$ for $\mathrm{S}+\mathrm{S}$ and to $\chi^{2} / \mathrm{DOF}=6.9 / 15$ for $\mathrm{Pb}+\mathrm{Pb}$.

We see here the breakdown of the concept of relative strangeness equilibrium [7] which is violated by various strange particle species. The thermal fit has the tendency to underestimate the meson yields, and the thermal $|S|=1$ and $|S|=2$ (anti)baryons are roughly in agreement with RQMD, while the $|S|=3$ (anti)baryons are overpredicted by the thermal model with optimized parameters relative to RQMD. The decrease of $\gamma_{s}$ from the thermal fit with a reduced number of hadrons compared to the fit with all stable hadrons is a result of the above-mentioned undersaturation of the RQMD multistrange hadrons. This can nicely be seen in Figs. 1 1 and 2.

An explanation for the above findings was offered in [27] where the unexpectedly small inverse slope [28] of the $\Omega p_{T}$ spectrum was investigated within the RQMD model. It was found that the $\Omega$ decouples very early from the rest of the fireball. It is therefore very unlikely that the $\Omega$ comes into chemical equilibrium with the rest of the hadrons. The same may also partly be true for the $\Xi$. 
A word of caution is necessary here. We see in Figs. 1 and 2 that the $\Omega$ and $\bar{\Omega}$ have the largest deviations from the thermal fit. However, one must note that the physics of the $\Omega(\bar{\Omega})$ production in RQMD is not treated on the same high quality level as the other particles. Since the $\Omega$ was of minor physical relevance before - this has changed with the high precision measurement of $\Omega$ ratios [30] and slopes [28] - a precise implementation of the $\Omega$ production physics in RQMD was neglected. The used strangeness suppression factors and decuplet-octet suppression factors result already in $e^{+} e^{-}$collisions in $\Omega$ and $\bar{\Omega}$ yields which are a factor of 2 too low. So far no $\Omega(\bar{\Omega})$ production via resonance states has been incorporated. The above-mentioned shortcomings reduce the $\Omega(\bar{\Omega})$ yields. Therefore an improvement of the $\Omega$ physics within RQMD will lead to smaller deviations from the thermal fit than seen now.

We conclude from this section that a relatively small subset of hadrons looks more thermal than the complete set of stable particles. Thermal behavior should therefore always be tested taking as many hadron species into account as possible. Nevertheless, we saw only minor changes in the thermal parameters between the thermal fit with a reduced number of hadrons and the thermal fit with all stable hadrons. Therefore, the experimentally accessible subset of hadrons is suited to determine the thermal parameters temperature, chemical potentials and the fireball point particle volume. $\gamma_{s}$, however, is sensitive (at least in the case of RQMD) to the multistrange (anti)baryons and should therefore be extracted by considering the full set of particles.

\section{THERMAL FITS INCLUDING RAPIDITY CUTS}

Particle yields or ratios are very often only accessible in a limited kinematic range. While an extrapolation in $p_{T}$ can be performed rather reliably due to the observed nearly exponential behavior of the $m_{T}$ spectra, an extrapolation in rapidity is much more difficult because different hadron species are known to have quite differently shaped rapidity spectra. A thermal fit in case of kinematic cuts needs model assumptions about the momentum distributions of the particles. The simplest assumption one can make is the assumption of Bjorken scaling in rapidity [31]. However, as a result of the much too low beam energy, Bjorken scaling has not been seen in RQMD or in the experimental data at SPS energies. Taking, however, the analyzed rapidity window to be very narrow around midrapidity the Bjorken scaling assumption might be approximately valid. In [32] it is shown that within the hydrodynamic model the extraction of thermal parameters from yields in a limited rapidity range works quite reasonably. We like to investigate the systematic error of rapidity cuts in a similar way with the help of RQMD events.
For this investigation we first integrate the particle momentum distribution over various rapidity intervals (see, e.g., Table ( ). Then we assume Bjorken scaling around midrapidity in the sense that these yields or ratios are fit-

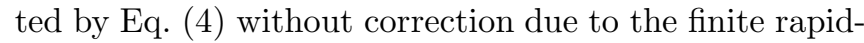
ity interval. Therefore the fitting procedure is the same as for the full momentum space yields, but the volume has to be considered as the sum of comoving subvolumes.

We show in Tables IV and VII the results for central $\mathrm{S}+\mathrm{S}$ collisions and in Tables $\mathrm{V}$ and VIII the corresponding results for $\mathrm{Pb}+\mathrm{Pb}$ collision. In $\mathrm{S}+\mathrm{S}$ collisions we see a clear decrease of the temperature with decreasing width of the rapidity window which is accompanied by an increase in $\gamma_{s}$ and a decrease in $\lambda_{u, d}$. The decrease of $\lambda_{u, d}$ is a result of the baryon hole around midrapidity in $\mathrm{S}+\mathrm{S}$ collisions 33 . The increase in $\gamma_{s}$ reflects the fact that RQMD, like the experiment, produces strange quarks more efficiently in the central region. On a weaker scale the same tendencies are seen for $\mathrm{Pb}+\mathrm{Pb}$ collisions.

An interpretation of this result is done in connection with a similar study of $\mathrm{S}+\mathrm{S}$ collisions within the hydrodynamic model [32]. In this study much smaller deviations between a global thermal fit and particle yields resulting from integration over different rapidity windows were found. In order to understand the difference one has to realize that in RQMD the physics, especially the physics of strange particle production, in the central region is different from the fragmentation regions. Therefore the change here in thermal parameters with the analyzed rapidity window resembles mostly a change in physics, while in the case of the hydrodynamical model by construction the pure kinematic bias was investigated. While a purely kinematic influence increases the temperature in the central region [32] the differences in physics decrease the central temperature compared to a $4 \pi$ analysis. It was further found [32] that for systems with large longitudinal flow the artificial change of thermal parameters is small. Therefore the change of thermal parameters with the rapidity window in the case of RQMD is clearly dominated by the physics component.

Thus we interpret the large change of thermal parameters with different rapidity window size as a result of no global equilibrium in these collisions and the deviations from a global equilibrium is much larger in $\mathrm{S}+\mathrm{S}$ collisions compared to $\mathrm{Pb}+\mathrm{Pb}$ collisions. However, there might still be local thermal and chemical equilibrium in the experiments as well as in the RQMD model. For a study of local equilibrium in RQMD we refer to 34. and in UrQMD to [35]. We only want here to emphasize that rapidity cuts can lead to considerable changes in the thermal parameters adjusted to a collision system. The change is the larger the further away the system is from global equilibrium. In addition we see that the $\chi^{2} / \mathrm{DOF}$ is best for $4 \pi$ integrated input data; as the rapidity interval is narrowed, we see a fluctuating but increasing $\chi^{2} / \mathrm{DOF}$. 


\section{CONCLUSIONS}

We have used the RQMD model to address some questions arising in the application of the thermal model to particle production in ultrarelativistic heavy ion collisions. We have performed a thermal fit to RQMD multiplicities, where the errors in the RQMD yields have been adjusted to be of the same order as typical for experiments. First we found that for an input consisting of only a small number of hadron species - typical for existing experiments - the RQMD yields can be nicely reproduced by a thermal model. This suggests at a first glance global thermal behavior. However, comparing the thermal model with the full set of stable particles leads to significant deviations. The largest discrepancy from thermal behavior is found for the multistrange hadrons. The usual small sample of particle species still gives nearly the same thermal parameters as the fit with the full set of stable hadrons. Experimentally the $4 \pi$ data are very scare and extrapolation is always required when a thermal fit is performed. Our results imply that one must be careful when using the thermal model to analyze the experimental data since the constrain is global, not local 36.

It is difficult to assess whether the complete set of $4 \pi$ multiplicities including the multistrange (anti)particles from RQMD shows still "good" thermal behavior. A judgment on the basis of the absolute value of $\chi^{2} / \mathrm{DOF}$ is not reasonable because $\chi^{2} / \mathrm{DOF}$ depends strongly on the assumed error of the multiplicities and, given certain existing deviations of the RQMD output from thermal behavior, becomes arbitrarily large as the statistical error on the yields becomes smaller and smaller by generating more and more events. A more useful criterion for judging the quality of the thermal fits may be given by the average deviation of the best thermal fit (with the relative errors from experiment) from the RQMD mean multiplicity. It is of the order of $20 \%$ for the fit in Tables IV and $\mathrm{V}$, of order $8 \%$ for a fit excluding the $\Omega$ and $\bar{\Omega}$, and of order $4 \%$ for a fit excluding $\Omega$, all $\Xi$ states and the corresponding antiparticles. Given that the absolute yields of the different hadrons species vary over three orders of magnitude these numbers suggest that RQMD yields are roughly in agreement with a thermal model interpretation. However, it must be shown - similar to the studies for the kinetic freeze-out in RQMD 34 or as done for the UrQMD model in [35] - that the extracted chemical freeze-out parameters are meaningful in the sense that the microscopic state of RQMD at chemical freeze-out is in agreement with chemical and kinetic equilibrium and that the resulting microscopic thermal parameters are the same as for the analysis presented here.

In a very recent publication the result of a thermal fit to UrQMD midrapidity ratios was presented [37]. In the case of $\mathrm{Pb}+\mathrm{Pb} 158 A \mathrm{GeV}$ collisions an agreement was found between the UrQMD multiplicities and the ther- mal model on the same quality level as presented here but with slightly different parameters for temperature $140 \mathrm{MeV}$ 37 vs $154 \mathrm{MeV}$ here (see Table VIII left column) and $\mu_{B} / T=1.50$ [37] vs 1.57 here. The detailed microscopic study in [37] revealed that two fundamental assumptions of the "thermal model," i.e., universal freeze-out and global equilibrium, are violated; from this the authors [37] concluded that thermal fits to particle ratios are meaningless. We, on the other hand, would like to reserve judgement on the meaningfulness of thermal fits to particle multiplicities until we understand why a thermal fit to particle yields works much better than the large violation of common freeze-out 27,37 and global equilibrium 3735 would suggest.

We also investigated the influence of rapidity cuts on the extracted thermal parameters. There is a change of thermal parameters with decreasing size of the analyzed rapidity window. This change is dominantly due to nonglobal equilibrium effects in these collisions. Thus one has to keep in mind that there is a nonnegligible bias on the extracted thermal parameters due to cuts in rapidity.

\section{Acknowledgments}

We would like to thank S.A. Bass for fruitful discussions. J.S., U.H., and N.X. acknowledge the hospitality of the INT (Seattle) where part of the work was done. The work was supported by BMBF, DFG, and GSI.

[1] Proceedings of "Quark Matter 97,", edited by T. Hatsuda, Y. Miake, S. Nagamiya, and K. Yagi [Nucl. Phys. A638 (1998)]; Proceedings of "Quark Matter 96,", edited by P. Braun-Munzinger, H.J. Specht, R. Stock, and H. Stöcker [ibid. A610 (1996)].

[2] J. Sollfrank, J. Phys. G 23, 1903 (1997).

[3] J. Sollfrank, M. Gaździcki, U. Heinz, and J. Rafelski Z. Phys. C 61, 659 (1994).

[4] J. Cleymans and A. Muronga, Phys. Lett. B 388, 5 (1996).

[5] F. Becattini, M. Gaździcki, and J. Sollfrank, Eur. Phys. J. C 5, 143 (1998).

[6] G.D. Yen, M.I. Gorenstein, W. Greiner, and S.N. Yang, Phys. Rev. C 56, 2210 (1997); G.D. Yen and M.I. Gorenstein, nucl-th/9808012.

[7] J. Rafelski, Phys. Lett. B 262, 333 (1991).

[8] J. Letessier and J. Rafelski, hep-ph/9807346; J. Letessier and J. Rafelski, Phys. Rev. C (to be published), hep$\mathrm{ph} / 9806386$.

[9] P. Braun-Munzinger, J. Stachel, J.P. Wessels, and N. Xu Phys. Lett. B 344, 43 (1995); Phys. Lett. B 365, 1 (1996).

[10] NA49 Collaboration, S.V. Afanasjev et al., Nucl. Phys. A610, 188c (1996).

[11] NA44 Collaboration, H. Bøggild et al., Nucl. Phys. A590, 215c (1995).

[12] WA97 Collaboration, G. Alexeev et al., Nucl. Phys. 
A590, 139c (1995).

[13] NA52 Collaboration, G. Ambrosini et al., J. Phys. G 23, 2135 (1997).

[14] H. Sorge, Phys. Rev. C 52, 3291 (1995).

[15] F. Becattini and U. Heinz, Z. Phys. C 76, 269 (1997).

[16] C. Slotta, J. Sollfrank, and U. Heinz, in Strangeness in Hadronic Matter, edited by J. Rafelski, AIP Conf. Proc. 340 (AIP Press, Woodbury, 1995), p. 462.

[17] R.M. Barnett et al., Phys. Rev. D 54, 1 (1996).

[18] NA35 Collaboration, T. Alber et al., Eur. Phys. J. C 2, 643 (1998)

[19] NA35 Collaboration, J. Bächler et al., Z. Phys. C 58, 367 (1993).

[20] NA35 Collaboration, T. Alber et al., Z. Phys. C 64, 195 (1994).

[21] NA35 Collaboration, T. Alber et al., Phys. Lett. B 366, 56 (1996).

[22] I. Huang Ph.D. thesis, University of Carlifornia at Davis, 1997.

[23] NA49 Collaboration, V. Friese et al., J. Phys. G 23, 1837 (1997).

[24] NA49 Collaboration, C. Bormann et al., J. Phys. G 23, 1817 (1997).

[25] H. Sorge, M. Berenguer, H. Stöcker, and W. Greiner,
Phys. Lett. B 289, 6 (1992).

[26] H. Sorge, Phys. Lett. B 402, 251 (1997); H. Sorge, Nucl. Phys. A630, 522 (1998).

[27] H. van Hecke, H. Sorge, and N. Xu, Phys. Rev. Lett. 81, 5764 (1998).

[28] WA97 Collaboration, E. Andersen et al., Phys. Lett. B 433, 209 (1998).

[29] WA97 Collaboration, (private communication).

[30] WA97 Collaboration, H.K. Holme et al., J. Phys. G 23, 1851 (1997).

[31] J.D. Bjorken, Phys. Rev. D 27, 140 (1983).

[32] J. Sollfrank, Eur. Phys. J. C (to be published), nuclth/9811078

33 NA35 Collaboration, J. Bächler et al., Phys. Rev. Lett. 72, 1419 (1994).

[34] H. Sorge, Phys. Lett. B 373, 16 (1996).

[35] L.V. Bravina et al., J. Phys. G (to be published), nuclth/9810036 L.V. Bravina et al., Phys. Lett. B 434, 379 (1998).

[36] Y. Pang, A. Sakaguchi, and N. Xu, (unpublished).

[37] S.A. Bass, H. Weber, C. Ernst, M. Bleicher, M. Belkacem, L. Bravina, S. Soff, H. Stöcker, W. Greiner, and C. Spieles, nucl-th/9810077; S.A. Bass et al., Phys. Rev. Lett. 81, 4092 (1998). 
TABLE I. Comparison between fitted and measured hadron abundances and ratios. Both thermal model calculations include the weak/electromagnetic decay of $\Sigma^{0}, \Xi^{0}, \Xi^{-}, \Omega$ and the corresponding antiparticles.

\begin{tabular}{|c|c|c|c|c|}
\hline Hadron & Measured & Ref. & Fitted in 5 & Grand canonical fit \\
\hline \multicolumn{5}{|c|}{$\mathrm{S}+\mathrm{S}$ collisions } \\
\hline$\overline{\mathrm{h}^{-}\left({ }^{a}\right)}$ & $98 \pm 3$ & $\mid 18$ & 92.63 & $\begin{array}{l}94.21 \\
\end{array}$ \\
\hline $\mathrm{K}^{+}$ & $12.5 \pm 0.4$ & 19 & 12.68 & 12.65 \\
\hline $\mathrm{K}_{s}^{0}$ & $10.5 \pm 1.7$ & 20 & 9.939 & 9.72 \\
\hline$\Lambda\left({ }^{b}\right)$ & $9.4 \pm 1.0$ & 20 & 7.692 & 8.30 \\
\hline $\bar{\Lambda}\left({ }^{b}\right)$ & $2.2 \pm 0.4$ & 20 & 1.474 & 1.633 \\
\hline$\overline{\mathrm{p}}\left({ }^{d}\right)$ & $1.15 \pm 0.4$ & 21 & 2.092 & 2.044 \\
\hline \multicolumn{5}{|c|}{$\mathrm{Pb}+\mathrm{Pb}$ collisions } \\
\hline Net baryon & $372 \pm 10$ & 22 & 375.7 & 374.3 \\
\hline $\mathrm{h}^{-}\left({ }^{a}\right)$ & $680 \pm 50$ & 10 & 650.2 & 661.0 \\
\hline $\mathrm{K}_{s}^{0}$ & $68 \pm 10$ & 10 & 58.27 & 58.22 \\
\hline$\phi$ & $5.4 \pm 0.7$ & 23 & 5.759 & 5.660 \\
\hline \multicolumn{5}{|c|}{$\begin{array}{l}a \text { - Defined as } \pi^{-}+\mathrm{K}^{-}+\overline{\mathrm{p}} . \\
b \text { - Includes feeding from } \Xi . \\
c \text { - Measured with the '+' }+ \text { '-' method, in this case limited } \\
\text { rapidity acceptance }(0.2-5.8) \text { to exclude spectators. } \\
d \text { - Measured in a restricted rapidity interval and extrapolated. } \\
\text { by assuming that } \overline{\mathrm{p}} \text { has the same rapidity distribution as the } \bar{\Lambda} \text {. }\end{array}$} \\
\hline
\end{tabular}

TABLE II. Hadron gas model fitted parameters. The fit itself is shown in Table [- The entries denoted by an asterisk are derived quantities while the others are fit parameters used by MINUIT.

\begin{tabular}{|c|c|c|c|c|}
\hline \multirow[b]{2}{*}{ parameter } & \multicolumn{2}{|c|}{$\mathrm{S}+\mathrm{S}$} & \multicolumn{2}{|c|}{$\mathrm{Pb}+\mathrm{Pb}$} \\
\hline & ref. 5 & grand canonical & ref. 阿 & grand canonical \\
\hline$T(\mathrm{MeV})$ & $\frac{4}{182.4 \pm 9.2}$ & $187.7 \pm 1.4$ & $192.6 \pm 8.1$ & $188.8 \pm 1.1$ \\
\hline$V T^{3} e^{-0.7 \mathrm{GeV} / T}$ & $3.51 \pm 0.15$ & $3.42^{*}$ & $24.3 \pm 1.6$ & $24.4^{*}$ \\
\hline$V\left(\mathrm{fm}^{3}\right)$ & $206^{*}$ & $166 \pm 11$ & $992^{*}$ & $1134 \pm 76$ \\
\hline$\gamma_{s}$ & $0.732 \pm 0.038$ & $0.731 \pm 0.033$ & $0.616 \pm 0.043$ & $0.620 \pm 0.047$ \\
\hline$\mu_{B} / T$ & $1.248 \pm 0.074$ & $1.251^{*}$ & $1.207 \pm 0.071$ & $1.258^{*}$ \\
\hline$\lambda_{u}$ & - & $1.516 \pm 0.021$ & - & $1.469 \pm 0.018$ \\
\hline$\lambda_{d}$ & - & $1.518 \pm 0.021$ & - & $1.547 \pm 0.022$ \\
\hline$\lambda_{s}$ & - & $1.069 \pm 0.005$ & - & $1.071 \pm 0.005$ \\
\hline$\chi^{2} / \mathrm{DOF}$ & $17.1 / 4$ & $12.0 / 4$ & $3.99 / 3$ & $2.33 / 3$ \\
\hline
\end{tabular}


TABLE III. List of relative errors of $4 \pi$ multiplicities and ratios used for RQMD multiplicities.

\begin{tabular}{|c|c|c|c|c|}
\hline \multirow[b]{2}{*}{ particle } & \multicolumn{2}{|c|}{$\mathrm{S}+\mathrm{S}$} & \multicolumn{2}{|c|}{$\mathrm{Pb}+\mathrm{Pb}$} \\
\hline & rel. error in \% & source & rel. error in \% & source \\
\hline $\mathrm{h}^{-}$ & 3.1 & 18 & 7.4 & 10 \\
\hline $\mathrm{p}-\overline{\mathrm{p}}$ & 6.1 & 18 & 12.9 & 10 \\
\hline net baryon & - & - & 2.7 & 22 \\
\hline $\mathrm{K}^{+} / \mathrm{K}^{-}$ & - & - & 5.6 & 24 \\
\hline $\bar{\Lambda} / \Lambda$ & - & - & 20.0 & 24 \\
\hline $\mathrm{p}$ & 6.1 & same as $\mathrm{p}-\overline{\mathrm{p}}$ & 12.9 & same as $\mathrm{p}-\overline{\mathrm{p}}$ \\
\hline$\overline{\mathrm{p}}$ & 34.8 & 21 & 34.8 & same as $\mathrm{S}+\mathrm{S}$ \\
\hline $\mathrm{n}$ & 6.1 & same as $p$ & 12.9 & same as p \\
\hline$\overline{\mathrm{n}}$ & 34.8 & same as $\bar{p}$ & 34.8 & same as $\bar{p}$ \\
\hline$\pi^{+}$ & 3.1 & same as $\mathrm{h}^{-}$ & 7.4 & same as $\mathrm{h}^{-}$ \\
\hline$\pi^{-}$ & 3.1 & same as $\mathrm{h}^{-}$ & 7.4 & same as $\mathrm{h}^{-}$ \\
\hline$\pi^{0}$ & 3.1 & same as $\mathrm{h}^{-}$ & 7.4 & same as $\mathrm{h}^{-}$ \\
\hline $\mathrm{K}^{+}$ & 3.2 & 19 & 3.2 & same as $\mathrm{S}+\mathrm{S}$ \\
\hline $\mathrm{K}^{-}$ & 5.8 & 19 & 5.8 & same as $\mathrm{S}+\mathrm{S}$ \\
\hline $\mathrm{K}_{s}^{0}$ & 16.2 & 20 & 14.7 & 10 \\
\hline$\phi$ & 13.0 & same as $\mathrm{Pb}+\mathrm{Pb}$ & 13.0 & 23 \\
\hline$\Lambda$ & 10.6 & 20 & 10.6 & same as $\mathrm{S}+\mathrm{S}$ \\
\hline $\bar{\Lambda}$ & 18.2 & 20 & 18.2 & same as $\mathrm{S}+\mathrm{S}$ \\
\hline$\Sigma^{+}$ & 10.6 & same as $\Lambda$ & 10.6 & same as $\Lambda$ \\
\hline $\bar{\Sigma}^{+}$ & 18.2 & same as $\bar{\Lambda}$ & 18.2 & same as $\bar{\Lambda}$ \\
\hline$\Sigma^{0}$ & 10.6 & same as $\Lambda$ & 10.6 & same as $\Lambda$ \\
\hline $\bar{\Sigma}^{0}$ & 18.2 & same as $\bar{\Lambda}$ & 18.2 & same as $\bar{\Lambda}$ \\
\hline$\Sigma^{-}$ & 10.6 & same as $\Lambda$ & 10.6 & same as $\Lambda$ \\
\hline $\bar{\Sigma}^{-}$ & 18.2 & same as $\bar{\Lambda}$ & 18.2 & same as $\bar{\Lambda}$ \\
\hline$\Xi^{-}$ & 10.0 & same as $\mathrm{Pb}+\mathrm{Pb}$ & 10.0 & $28\left({ }^{a}\right)$ \\
\hline $\bar{\Xi}^{-}$ & 15.0 & same as $\mathrm{Pb}+\mathrm{Pb}$ & 15.0 & $29\left({ }^{b}\right)$ \\
\hline$\Xi^{0}$ & 10.0 & same as $\Xi^{-}$ & 10.0 & same as $\Xi^{-}$ \\
\hline $\bar{\Xi}^{0}$ & 15.0 & same as $\bar{\Xi}^{-}$ & 15.0 & same as $\bar{\Xi}^{-}$ \\
\hline$\Omega^{-}$ & 23.0 & same as $\mathrm{Pb}+\mathrm{Pb}$ & 23.0 & $28\left({ }^{a}\right)$ \\
\hline $\bar{\Omega}^{-}$ & 34.5 & same as $\mathrm{Pb}+\mathrm{Pb}$ & 34.5 & $29\left({ }^{b}\right)$ \\
\hline $\begin{array}{l}\left.{ }^{a}\right) \text { We assu } \\
\text { per ever } \\
\left({ }^{b}\right) \text { The rela } \\
50 \% \text { lar }\end{array}$ & $\begin{array}{l}\text { relative error } \\
\text { ematic window } \\
\text { multiplicities }\end{array}$ & $\begin{array}{l}\text { as the error on } n \\
\text { or the most centr } \\
\text { ange antibaryons }\end{array}$ & & \\
\hline
\end{tabular}


TABLE IV. RQMD(version 2.3) multiplicities of $200 A \mathrm{GeV} / \mathrm{c} \mathrm{S}+\mathrm{S}$ collisions. Trigger cross section $\sigma_{\text {trig }} \leq 5 \% \sigma_{\text {geom }}(b \leq 0.85$ fm).

\begin{tabular}{|c|c|c|c|c|c|c|c|c|c|c|}
\hline & \multicolumn{2}{|c|}{$|y| \leq 0.5$} & \multicolumn{2}{|c|}{$|y| \leq 1.0$} & \multicolumn{2}{|c|}{$|y| \leq 1.5$} & \multicolumn{2}{|c|}{$|y|<2.0$} & \multicolumn{2}{|c|}{$4 \pi$} \\
\hline & RQMD & therm. & RQMD & therm. & RQMD & therm. & RQMD & therm. & RQMD & therm. \\
\hline$p$ & $3.13 \pm 0.19$ & 3.297 & $6.95 \pm 0.42$ & 7.304 & $11.65 \pm 0.71$ & 11.954 & $16.47 \pm 1.00$ & 16.624 & $24.24 \pm 1.47$ & $\overline{23.552}$ \\
\hline $\bar{p}$ & $0.50 \pm 0.17$ & 0.342 & $0.99 \pm 0.34$ & 0.975 & $1.25 \pm 0.43$ & 1.122 & $1.36 \pm 0.47$ & 1.187 & $1.40 \pm 0.48$ & 1.301 \\
\hline$n$ & $3.20 \pm 0.19$ & 3.292 & $7.23 \pm 0.44$ & 7.296 & $11.79 \pm 0.71$ & 11.943 & $16.60 \pm 1.01$ & 16.611 & $24.23 \pm 1.47$ & 23.536 \\
\hline $\bar{n}$ & $0.49 \pm 0.17$ & 0.340 & $0.89 \pm 0.30$ & 0.972 & $1.12 \pm 0.39$ & 1.119 & $1.23 \pm 0.42$ & 1.183 & $1.27 \pm 0.44$ & 1.297 \\
\hline$\pi^{+}$ & $18.42 \pm 0.57$ & 18.719 & $35.78 \pm 1.10$ & 36.959 & $50.36 \pm 1.56$ & 51.527 & $60.89 \pm 1.88$ & 62.484 & $72.54 \pm 2.24$ & 74.863 \\
\hline$\pi^{-}$ & $18.80 \pm 0.58$ & 18.732 & $36.10 \pm 1.11$ & 36.983 & $50.56 \pm 1.56$ & 51.564 & $60.18 \pm 1.86$ & 62.532 & $72.69 \pm 2.25$ & 74.919 \\
\hline$\pi^{0}$ & $19.18 \pm 0.59$ & 20.562 & $37.15 \pm 1.15$ & 40.267 & $52.31 \pm 1.62$ & 56.141 & $63.38 \pm 1.96$ & 67.837 & $75.31 \pm 2.33$ & 80.891 \\
\hline$K^{+}$ & $3.34 \pm 0.10$ & 3.136 & $6.40 \pm 0.20$ & 5.477 & $8.92 \pm 0.28$ & 8.018 & $10.51 \pm 0.33$ & 9.384 & $11.45 \pm 0.36$ & 10.354 \\
\hline$K^{-}$ & $2.10 \pm 0.12$ & 2.152 & $3.86 \pm 0.22$ & 3.702 & $5.16 \pm 0.29$ & 4.988 & $5.91 \pm 0.34$ & 5.469 & $6.26 \pm 0.36$ & 5.567 \\
\hline$K_{s}^{0}$ & $2.70 \pm 0.43$ & 2.623 & $5.08 \pm 0.82$ & 4.563 & $6.98 \pm 1.13$ & 6.467 & $8.11 \pm 1.31$ & 7.386 & $8.76 \pm 1.41$ & 7.921 \\
\hline$\phi$ & $0.42 \pm 0.054$ & 0.309 & $0.81 \pm 0.10$ & 0.519 & $1.06 \pm 0.13$ & 0.754 & $1.17 \pm 0.15$ & 0.811 & $1.19 \pm 0.15$ & 0.790 \\
\hline$\Lambda$ & $1.05 \pm 0.11$ & 0.838 & $2.21 \pm 0.23$ & 1.663 & $3.32 \pm 0.35$ & 2.688 & $4.18 \pm 0.44$ & 3.416 & $4.72 \pm 0.50$ & 4.200 \\
\hline $\bar{\Lambda}$ & $0.25 \pm 0.045$ & 0.131 & $0.44 \pm 0.080$ & 0.343 & $0.56 \pm 0.10$ & 0.429 & $0.60 \pm 0.10$ & 0.447 & $0.61 \pm 0.11$ & 0.469 \\
\hline$\Sigma^{+}$ & $0.50 \pm 0.053$ & 0.329 & $0.96 \pm 0.10$ & 0.650 & $1.49 \pm 0.15$ & 1.050 & $1.84 \pm 0.19$ & 1.333 & $2.11 \pm 0.22$ & 1.636 \\
\hline $\bar{\Sigma}^{+}$ & $0.10 \pm 0.018$ & 0.051 & $0.17 \pm 0.030$ & 0.134 & $0.23 \pm 0.041$ & 0.168 & $0.25 \pm 0.045$ & 0.175 & $0.25 \pm 0.045$ & 0.183 \\
\hline$\Sigma^{0}$ & $0.43 \pm 0.045$ & 0.325 & $0.82 \pm 0.086$ & 0.643 & $1.29 \pm 0.13$ & 1.039 & $1.59 \pm 0.16$ & 1.320 & $1.77 \pm 0.18$ & 1.620 \\
\hline $\bar{\Sigma}^{0}$ & $0.11 \pm 0.020$ & 0.050 & $0.19 \pm 0.034$ & 0.133 & $0.24 \pm 0.043$ & 0.166 & $0.26 \pm 0.047$ & 0.173 & $0.26 \pm 0.047$ & 0.181 \\
\hline$\Sigma^{-}$ & $0.47 \pm 0.049$ & 0.319 & $0.94 \pm 0.099$ & 0.633 & $1.39 \pm 0.14$ & 1.023 & $1.74 \pm 0.18$ & 1.300 & $1.92 \pm 0.20$ & 1.597 \\
\hline $\bar{\Sigma}^{-}$ & $0.11 \pm 0.020$ & 0.049 & $0.19 \pm 0.034$ & 0.130 & $0.24 \pm 0.043$ & 0.163 & $0.26 \pm 0.047$ & 0.170 & $0.26 \pm 0.047$ & 0.179 \\
\hline$\Xi^{0}$ & $0.11 \pm 0.011$ & 0.135 & $0.25 \pm 0.025$ & 0.237 & $0.36 \pm 0.036$ & 0.378 & $0.43 \pm 0.043$ & 0.439 & $0.44 \pm 0.044$ & 0.466 \\
\hline $\bar{\Xi}^{0}$ & $0.07 \pm 0.010$ & 0.032 & $0.12 \pm 0.018$ & 0.075 & $0.15 \pm 0.022$ & 0.102 & $0.16 \pm 0.024$ & 0.105 & $0.16 \pm 0.024$ & 0.105 \\
\hline$\Xi^{-}$ & $0.14 \pm 0.014$ & 0.133 & $0.26 \pm 0.026$ & 0.233 & $0.36 \pm 0.036$ & 0.372 & $0.43 \pm 0.043$ & 0.432 & $0.44 \pm 0.044$ & 0.459 \\
\hline $\bar{\Xi}^{-}$ & $0.06 \pm 0.009$ & 0.031 & $0.11 \pm 0.016$ & 0.074 & $0.13 \pm 0.019$ & 0.101 & $0.15 \pm 0.022$ & 0.103 & $0.15 \pm 0.022$ & 0.103 \\
\hline $\bar{\Omega}^{-}$ & $0.01 \pm 0.002$ & 0.016 & $0.01 \pm 0.002$ & 0.028 & $0.02 \pm 0.004$ & 0.044 & $0.02 \pm 0.004$ & 0.047 & $0.02 \pm 0.004$ & 0.044 \\
\hline $\bar{\Omega}^{-}$ & $0.01 \pm 0.003$ & 0.005 & $0.01 \pm 0.003$ & 0.014 & $0.01 \pm 0.003$ & 0.020 & $0.01 \pm 0.003$ & 0.021 & $0.01 \pm 0.003$ & 0.020 \\
\hline
\end{tabular}

TABLE V. RQMD(version 2.3) multiplicities of $158 A \mathrm{GeV} / \mathrm{c} \mathrm{Pb}+\mathrm{Pb}$ collisions. Trigger cross section $\sigma_{\text {trig }} \leq 5 \% \sigma_{\text {geom }}(b \leq 3$ fm).

\begin{tabular}{|c|c|c|c|c|c|c|c|c|c|c|}
\hline & \multicolumn{2}{|c|}{$|y| \leq 0.5$} & \multicolumn{2}{|c|}{$|y| \leq 1.0$} & \multicolumn{2}{|c|}{$|y| \leq 1.5$} & \multicolumn{2}{|c|}{$|y| \leq 2.0$} & \multicolumn{2}{|c|}{$4 \pi$} \\
\hline & RQMD & therm. & RQMD & therm. & RQMD & therm. & RQMD & therm. & RQMD & therm. \\
\hline$p$ & $27.06 \pm 3.49$ & 32.321 & $55.08 \pm 7.10$ & 64.884 & $82.51 \pm 10.6$ & 96.019 & $105.00 \pm 13.5$ & 120.752 & $131.40 \pm 17.0$ & $\overline{146.992}$ \\
\hline $\bar{p}$ & $1.68 \pm 0.58$ & 2.295 & $3.14 \pm 1.09$ & 4.011 & $3.92 \pm 1.36$ & 5.184 & $4.37 \pm 1.52$ & 5.746 & $4.58 \pm 1.59$ & 6.348 \\
\hline$n$ & $28.89 \pm 3.72$ & 33.513 & $58.00 \pm 7.48$ & 67.476 & $85.82 \pm 11.1$ & 100.184 & $109.35 \pm 14.1$ & 126.259 & $138.83 \pm 17.9$ & 154.054 \\
\hline $\bar{n}$ & $1.77 \pm 0.61$ & 2.203 & $3.25 \pm 1.13$ & 3.839 & $4.05 \pm 1.40$ & 4.946 & $4.45 \pm 1.54$ & 5.471 & $4.66 \pm 1.62$ & 6.030 \\
\hline$\pi^{+}$ & $144.16 \pm 10.7$ & 156.050 & $271.73 \pm 20.1$ & 291.882 & $369.94 \pm 27.4$ & 397.705 & $435.64 \pm 32.2$ & 470.565 & $500.05 \pm 37.0$ & 534.792 \\
\hline$\pi^{-}$ & $151.11 \pm 11.2$ & 163.344 & $284.80 \pm 21.1$ & 306.556 & $389.28 \pm 28.8$ & 419.270 & $460.63 \pm 34.1$ & 497.486 & $531.42 \pm 39.3$ & 567.035 \\
\hline$\pi^{0}$ & $152.56 \pm 11.3$ & 176.860 & $287.54 \pm 21.3$ & 331.296 & $391.99 \pm 29.0$ & 450.767 & $461.97 \pm 34.2$ & 532.048 & $531.37 \pm 39.3$ & 601.890 \\
\hline$K^{+}$ & $31.91 \pm 1.02$ & 30.007 & $60.16 \pm 1.92$ & 56.971 & $81.12 \pm 2.59$ & 76.911 & $93.69 \pm 2.99$ & 88.895 & $100.33 \pm 3.21$ & 95.780 \\
\hline$K^{-}$ & $18.76 \pm 1.08$ & 19.155 & $34.72 \pm 2.01$ & 35.180 & $45.55 \pm 2.64$ & 45.836 & $51.51 \pm 2.98$ & 51.683 & $54.28 \pm 3.14$ & 54.358 \\
\hline$K_{s}^{0}$ & $25.59 \pm 3.76$ & 24.488 & $47.92 \pm 7.04$ & 45.934 & $63.73 \pm 9.36$ & 61.239 & $72.75 \pm 10.7$ & 70.173 & $77.30 \pm 11.4$ & 75.0 \\
\hline$\phi$ & $4.55 \pm 0.59$ & 3.179 & $8.19 \pm 1.06$ & 5.999 & $10.74 \pm 1.39$ & 7.853 & $11.82 \pm 1.53$ & 8.654 & $12.08 \pm 1.57$ & 8.681 \\
\hline$\Lambda$ & $10.79 \pm 1.14$ & 8.936 & $20.63 \pm 2.18$ & 17.769 & $29.34 \pm 3.11$ & 25.326 & $35.38 \pm 3.75$ & 30.367 & $38.09 \pm 4.03$ & 34.206 \\
\hline $\bar{\Lambda}$ & $1.24 \pm 0.22$ & 1.019 & $2.06 \pm 0.37$ & 1.829 & $2.62 \pm 0.47$ & 2.367 & $2.90 \pm 0.52$ & 2.569 & $2.99 \pm 0.54$ & 2.696 \\
\hline$\Sigma^{+}$ & $4.30 \pm 0.45$ & 3.412 & $8.49 \pm 0.89$ & 6.770 & $12.20 \pm 1.29$ & 9.623 & $14.48 \pm 1.53$ & 11.518 & $15.32 \pm 1.62$ & 12.946 \\
\hline $\bar{\Sigma}^{+}$ & $0.55 \pm 0.10$ & 0.411 & $0.88 \pm 0.16$ & 0.740 & $1.12 \pm 0.20$ & 0.960 & $1.25 \pm 0.22$ & 1.043 & $1.27 \pm 0.23$ & 1.096 \\
\hline$\Sigma^{0}$ & $4.21 \pm 0.44$ & 3.465 & $8.50 \pm 0.90$ & 6.890 & $12.07 \pm 1.27$ & 9.817 & $14.35 \pm 1.52$ & 11.769 & $15.38 \pm 1.63$ & 13.250 \\
\hline $\bar{\Sigma}^{0}$ & $0.47 \pm 0.085$ & 0.395 & $0.83 \pm 0.15$ & 0.709 & $1.07 \pm 0.19$ & 0.918 & $1.17 \pm 0.21$ & 0.996 & $1.20 \pm 0.21$ & 1.045 \\
\hline$\Sigma^{-}$ & $4.45 \pm 0.47$ & 3.496 & $8.81 \pm 0.93$ & 6.967 & $12.35 \pm 1.30$ & 9.952 & $14.77 \pm 1.56$ & 11.949 & $15.76 \pm 1.67$ & 13.478 \\
\hline $\bar{\Sigma}^{-}$ & $0.49 \pm 0.089$ & 0.377 & $0.86 \pm 0.15$ & 0.676 & $1.14 \pm 0.20$ & 0.873 & $1.27 \pm 0.23$ & 0.945 & $1.28 \pm 0.23$ & 0.991 \\
\hline$\Xi^{0}$ & $1.57 \pm 0.15$ & 1.532 & $3.01 \pm 0.30$ & 3.008 & $4.13 \pm 0.41$ & 4.114 & $4.66 \pm 0.46$ & 4.696 & $4.78 \pm 0.47$ & 4.880 \\
\hline $\bar{\Xi}^{0}$ & $0.42 \pm 0.063$ & 0.295 & $0.72 \pm 0.10$ & 0.545 & $0.95 \pm 0.14$ & 0.707 & $1.04 \pm 0.15$ & 0.752 & $1.05 \pm 0.15$ & 0.751 \\
\hline$\Xi^{-}$ & $1.53 \pm 0.15$ & 1.528 & $3.04 \pm 0.30$ & 3.006 & $4.16 \pm 0.41$ & 4.118 & $4.71 \pm 0.47$ & 4.706 & $4.84 \pm 0.48$ & 4.896 \\
\hline $\bar{\Xi}^{-}$ & $0.41 \pm 0.061$ & 0.284 & $0.69 \pm 0.10$ & 0.524 & $0.92 \pm 0.13$ & 0.679 & $1.00 \pm 0.15$ & 0.722 & $1.00 \pm 0.15$ & 0.720 \\
\hline$\Omega^{-}$ & $0.10 \pm 0.023$ & 0.203 & $0.20 \pm 0.046$ & 0.396 & $0.26 \pm 0.059$ & 0.525 & $0.28 \pm 0.066$ & 0.571 & $0.28 \pm 0.066$ & 0.552 \\
\hline $\bar{\Omega}^{-}$ & $0.03 \pm 0.010$ & 0.064 & $0.06 \pm 0.020$ & 0.122 & $0.08 \pm 0.027$ & 0.160 & $0.08 \pm 0.027$ & 0.167 & $0.08 \pm 0.027$ & 0.159 \\
\hline
\end{tabular}


TABLE VI. Thermal parameters of fitting RQMD yields and ratios in $\mathrm{S}+\mathrm{S}$ (left) and $\mathrm{Pb}+\mathrm{Pb}$ (right) collisions. The same particles and ratios were taken like in the fit of [5]. The input data set was extracted from the entries in Tables IV] and $\mathrm{V}$, respectively. The relative error is as in Table 1 .

\begin{tabular}{|c|c|c|c|c|c|}
\hline \multirow[b]{2}{*}{ particle } & \multicolumn{2}{|c|}{$\mathrm{S}+\mathrm{S}$} & \multirow[b]{2}{*}{ particle } & \multicolumn{2}{|c|}{$\mathrm{Pb}+\mathrm{Pb}$} \\
\hline & RQMD & thermal & & RQMD & thermal \\
\hline$h^{-}$ & $82.98 \pm 2.57$ & 83.77 & $h^{-}$ & $612.5 \pm 45.32$ & 598.87 \\
\hline$K_{s}^{0}$ & $8.76 \pm 1.42$ & 8.74 & $K_{s}^{0}$ & $77.31 \pm 11.36$ & 81.71 \\
\hline$p-\bar{p}$ & $22.84 \pm 1.39$ & 22.22 & $p-\bar{p}$ & $126.82 \pm 16.36$ & 126.50 \\
\hline$K^{+}$ & $11.45 \pm 0.37$ & 11.53 & $\phi$ & $12.0 \pm 1.57$ & 11.88 \\
\hline$K^{-}$ & $6.26 \pm 0.36$ & 6.02 & $K^{+} / K^{-}$ & $1.847 \pm 0.104$ & 1.832 \\
\hline$\Lambda$ & $4.72 \pm 0.50$ & 4.82 & $\bar{\Lambda} / \Lambda$ & $0.0785 \pm 0.016$ & 0.080 \\
\hline $\bar{\Lambda}$ & $0.61 \pm 0.11$ & 0.597 & net baryon & $346.6 \pm 9.36$ & 347.19 \\
\hline $\bar{p}$ & $1.4 \pm 0.49$ & 1.38 & & & \\
\hline $\bar{\chi}^{2} / \mathrm{DOF}$ & & $0.83 / 4$ & & & $0.29 / 3$ \\
\hline$\overline{T(\mathrm{MeV})}$ & & $179.4 \pm 7.5$ & & & $156.7 \pm 8.6$ \\
\hline$V\left(\mathrm{fm}^{3}\right)$ & & $195 \pm 65$ & & & $3008 \pm 1780$ \\
\hline$\gamma_{s}$ & & $0.769 \pm 0.045$ & & & $1.129 \pm 0.17$ \\
\hline$\lambda_{u}$ & & $1.609 \pm 0.026$ & & & $1.677 \pm 0.044$ \\
\hline$\lambda_{d}$ & & $1.610 \pm 0.026$ & & & $1.761 \pm 0.065$ \\
\hline$\lambda_{s}$ & & $1.102 \pm 0.032$ & & & $1.208 \pm 0.059$ \\
\hline
\end{tabular}

TABLE VII. Thermal parameters of fitting RQMD multiplicities in various rapidity intervals for $\mathrm{S}+\mathrm{S}$ collisions (trigger cross section $\left.\sigma_{\text {trig }} \leq 5 \% \sigma_{\text {geom }}\right)$. The fit itself is shown in Table IV.

\begin{tabular}{cccccc}
\hline \hline & $|y| \leq 0.5$ & $|y| \leq 1.0$ & $|y| \leq 1.5$ & $|y| \leq 2.0$ & $4 \pi$ \\
\hline$T(\mathrm{MeV})$ & $153.3 \pm 1.3$ & $166.6 \pm 3.9$ & $167.5 \pm 2.7$ & $168.5 \pm 2.7$ & $261 \pm 30$ \\
$V\left(\mathrm{fm}^{3}\right)$ & $147 \pm 8$ & $173 \pm 29$ & $227 \pm 26$ & $0.748 \pm 0.018$ & $0.670 \pm 32$ \\
$\gamma_{s}$ & $0.905 \pm 0.027$ & $0.768 \pm 0.022$ & $0.793 \pm 0.019$ & $1.555 \pm 0.017$ & $1.623 \pm 0.019$ \\
$\lambda_{u}$ & $1.460 \pm 0.017$ & $1.400 \pm 0.016$ & $1.485 \pm 0.015$ & $1.556 \pm 0.017$ & $1.625 \pm 0.019$ \\
$\lambda_{d}$ & $1.461 \pm 0.017$ & $1.401 \pm 0.016$ & $1.487 \pm 0.015$ & $1.145 \pm 0.011$ & $1.138 \pm 0.013$ \\
$\lambda_{s}$ & $1.188 \pm 0.007$ & $1.124 \pm 0.010$ & $1.136 \pm 0.010$ & $111.91 / 21$ & $94.72 / 21$ \\
\hline$\chi^{2} / \mathrm{DOF}$ & $115.32 / 21$ & $152.20 / 21$ & $98.51 / 21$ & \\
\hline \hline
\end{tabular}

TABLE VIII. Thermal parameters of fitting RQMD multiplicities in various rapidity intervals for $\mathrm{Pb}+\mathrm{Pb}$ collisions (trigger cross section $\sigma_{\text {trig }} \leq 5 \% \sigma_{\text {geom }}$ ). The fit itself is shown in Table $\mathrm{V}$.

\begin{tabular}{|c|c|c|c|c|c|}
\hline & $|y| \leq 0.5$ & $|y| \leq 1.0$ & $|y| \leq 1.5$ & $|y| \leq 2.0$ & $4 \pi$ \\
\hline$\overline{T(\mathrm{MeV})}$ & $152.8 \pm 4.8$ & $153.3 \pm 3.3$ & $154.4 \pm 2.9$ & $154.4 \pm 2.7$ & $155.4 \pm 2.6$ \\
\hline$V\left(\mathrm{fm}^{3}\right)$ & $1223 \pm 277$ & $2215 \pm 347$ & $2868 \pm 390$ & $3386 \pm 427$ & $3706 \pm 460$ \\
\hline$\gamma_{s}$ & $1.019 \pm 0.046$ & $1.024 \pm 0.037$ & $1.000 \pm 0.033$ & $0.966 \pm 0.031$ & $0.900 \pm 0.029$ \\
\hline$\lambda_{u}$ & $1.538 \pm 0.032$ & $1.573 \pm 0.024$ & $1.608 \pm 0.022$ & $1.641 \pm 0.022$ & $1.667 \pm 0.022$ \\
\hline$\lambda_{d}$ & $1.595 \pm 0.041$ & $1.635 \pm 0.031$ & $1.676 \pm 0.028$ & $1.714 \pm 0.028$ & $1.745 \pm 0.028$ \\
\hline$\lambda_{s}$ & $1.211 \pm 0.017$ & $1.216 \pm 0.014$ & $1.219 \pm 0.014$ & $1.227 \pm 0.014$ & $1.230 \pm 0.014$ \\
\hline$\overline{\chi^{2} / D O F}$ & $79.62 / 21$ & $66.17 / 21$ & $67.38 / 21$ & $67.61 / 21$ & $55.61 / 21$ \\
\hline
\end{tabular}


FIG. 1. Thermal fit of RQMD $4 \pi$ yields of $\mathrm{S}+\mathrm{S}$ collisions. The solid squares denote the input yields of the reduced fit of Table VI. The open circles show the resulting other $4 \pi$ multiplicities of stable particles not included in the fit.

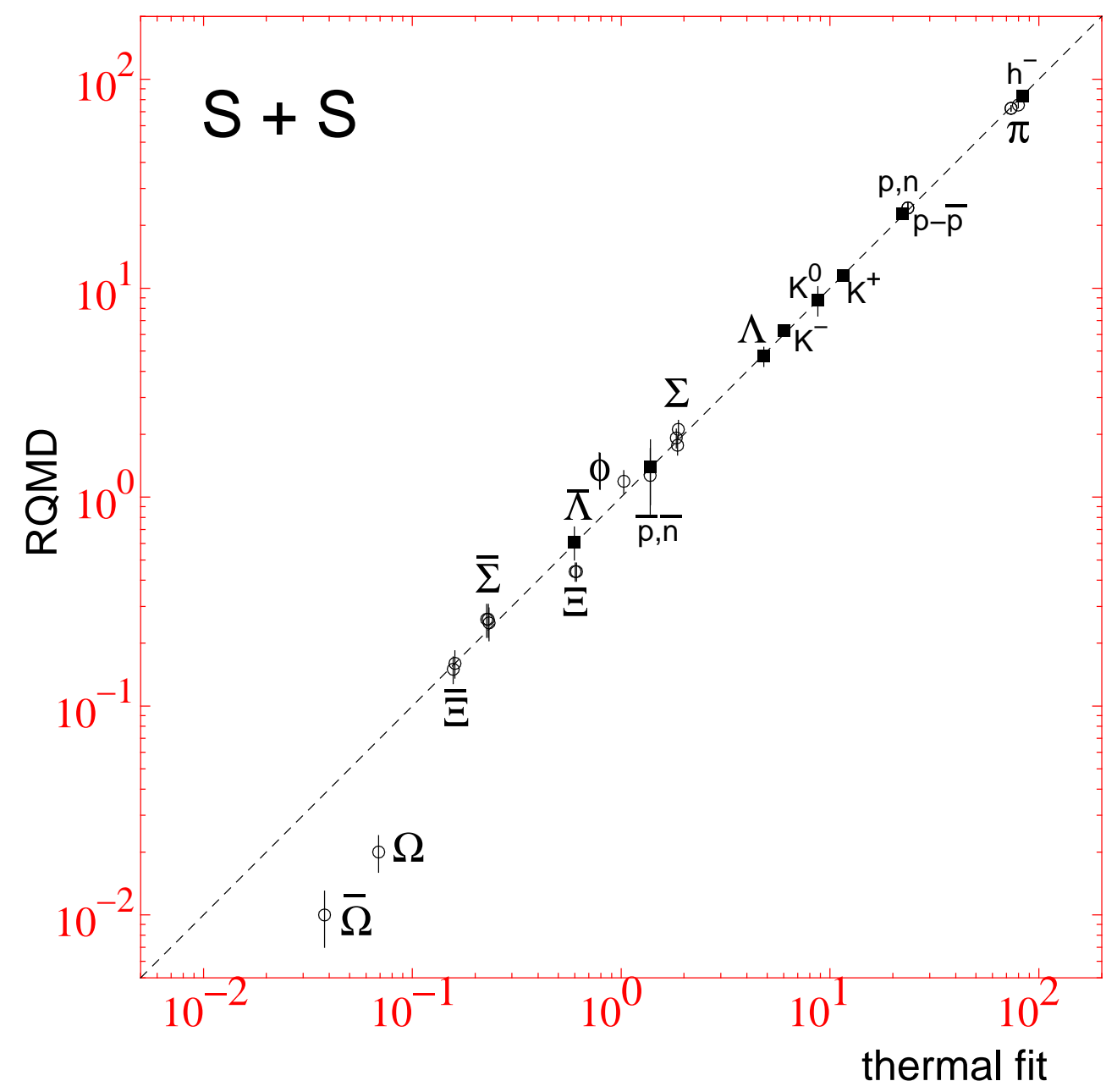


FIG. 2. Thermal fit of RQMD $4 \pi$ yields of $\mathrm{Pb}+\mathrm{Pb}$ collisions. The solid squares denote the input yields of the reduced fit of Table VI. The open circles show the resulting other $4 \pi$ multiplicities of stable particles not included in the fit.

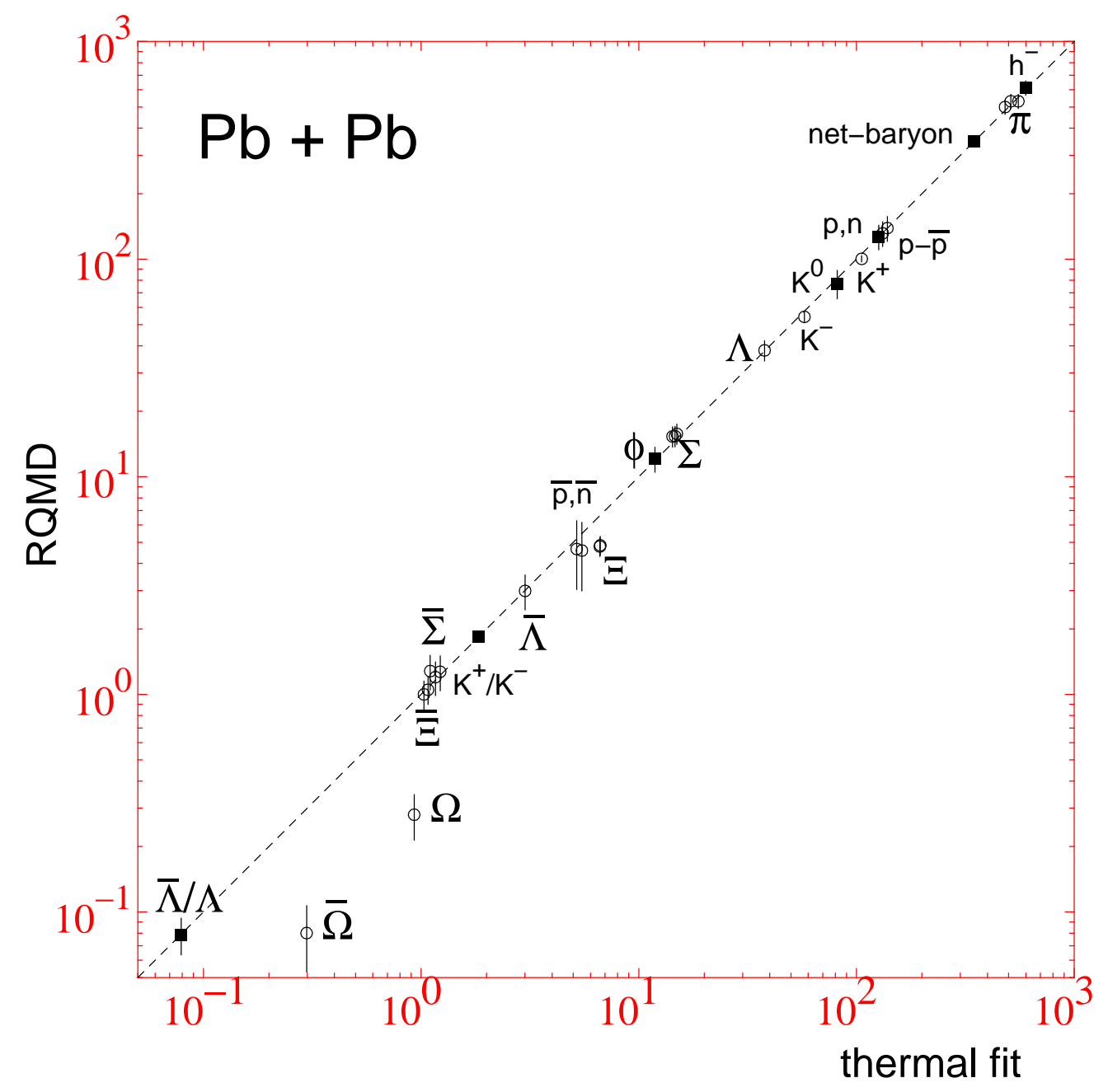

